\title{
Surface Preparation of InAs (110) Using Atomic Hydrogen
}

\author{
T.D. Veal ${ }^{*}$, C.F. McConville* and S.H. Al-Harthi ${ }^{* *}$
}

${ }^{*}$ Department of Physics, University of Warwick, CV4 7AL, United Kingdom, **Department of Physics, College of Science, Sultan Qaboos University, P.O.Box 36, Al khod 123, Muscat, Sultanate of Oman, ${ }^{* \star}$ Email: Salim1@squ.edu.om.
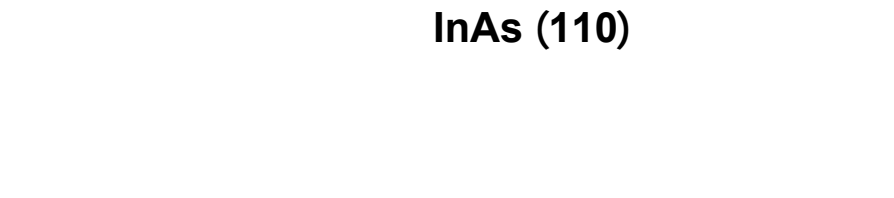

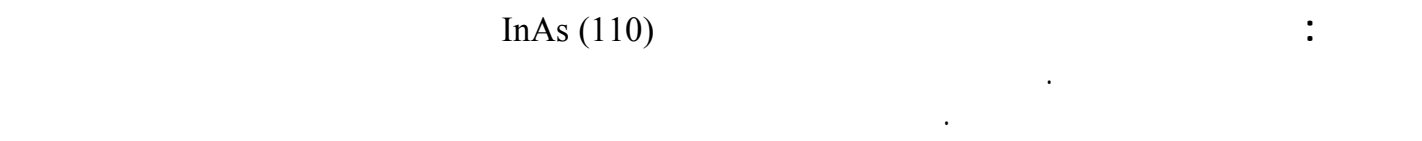

\begin{abstract}
Atomic hydrogen cleaning has been used to produce structurally and electronically damage-free InAs(110) surfaces. X-ray photoelectron spectroscopy (XPS) was used to obtain chemical composition and chemical state information about the surface, before and after the removal of the atmospheric contamination. Low energy electron diffraction (LEED) and high-resolution electron-energy-loss spectroscopy (HREELS) were also used, respectively, to determine the surface reconstruction and degree of surface ordering, and to probe the adsorbed contaminant vibrational modes and the collective excitations of the clean surface. Clean, ordered and stoichiometric InAs(110)-(1×1) surfaces were obtained by exposure to thermally generated atomic hydrogen at a substrate temperature as low as $400^{\circ} \mathrm{C}$. Semi-classical dielectric theory analysis of HREEL spectra of the phonon and plasmon excitations of the clean surface indicate that no electronic damage or dopant passivation were induced by the surface preparation method.
\end{abstract}

KEYWORDS: InAs(110), Hydrogen Cleaning, Plasmon Excitations, HREEL, XPS

\section{Introduction}

$\mathbf{T}$

he preparation of clean, ordered and stoichiometric III-V semiconductor surfaces is generally problematic without the extensive facilities available with molecular beam epitaxy (MBE). Even in an MBE-chamber, the thermal desorption of oxides in the presence of an arsenic partial pressure to prepare the surface of InAs is made difficult by the need for precise control of substrate temperature because the oxide and arsenic desorption temperatures are very close together (Schäfer et al 2000). When MBE facilities are unavailable, the standard method of in situ cleaning of polar InAs surfaces is cycles of low energy ion bombardment and annealing. However, this has been shown to cause severe structural damage, resulting in the introduction of defects, enhanced carrier concentrations and reduced mobilities within the outermost $1000 \AA$ of the material (Magnée et al 1995, Bell et al 1996a, Bell et al 1996b). Traditionally, the non-polar (110) surfaces of III-V materials have been produced by cleaving in vacuum, as this is the fracture plane of these zincblende materials. However, from cleave to cleave large variations in the surface step densities result. These steps have been observed using scanning tunnelling microscopy where (111), (112) and (110)-type steps have all been identified on InAs(110) (Liang et al 1993). The consequent modification of the surface, where the reactivity is likely to be dominated by the step density profile, will result in significant non-uniformity in the surface properties with each cleave. This is 
evident from photoemission studies, where the expected flat-band condition, with no electron accumulation, is rarely achieved for $\operatorname{InAs}(110)$. The surface electronic structure is extremely sensitive to the presence of cleave-induced steps, which result in downward band bending and accumulation layer formation (Karlsson et al 1998). An alternative surface preparation technique, atomic hydrogen cleaning (AHC), offers an effective way of obtaining clean, undamaged InAs(110) surfaces with uniform step densities. This method has previously been shown to be an efficient, relatively low temperature technique for obtaining clean, ordered and damage-free GaAs(001) and InP(001) surfaces (Kikawa et al 1994, Petit and Houzay 1992, Petit and Houzay 1994, Goto et al 1995).

AHC of the polar (100) and (111) surfaces of InAs has previously been studied using Auger electron spectroscopy (AES), high-resolution electron-energy-loss spectroscopy (HREELS) and low energy electron diffraction (LEED) (Bell et al 1997, Bell et al 1998). Whilst the removal of contamination by AHC was confirmed by AES, no chemical state information was available to indicate the reaction mechanisms that occur during AHC. Here, the use of x-ray photoelectron spectroscopy (XPS) before and after AHC, with some complementary use of HREELS as an adsorbate vibrational spectroscopy, permits the investigation of the processes by which AHC of $\operatorname{InAs}(110)$ proceeds. LEED was used to monitor the surface ordering before and after the cleaning process. HREELS was used to study the surface vibrational modes of the contaminants on the asloaded surfaces and collective excitations of the clean surface.

\section{Experimental Details}

Samples from S-doped $\operatorname{InAs}(110)$ wafers $\left(n \sim 4 \times 10^{18} \mathrm{~cm}^{-3}\right)$ were individually loaded into the HREELS spectrometer (base pressure $<5 \times 10^{-10} \mathrm{mbar}$ ) without any ex situ treatment. Molecular hydrogen was thermally cracked in an atomic hydrogen source (EPI Europe) as it passed over the W-filament held at $\sim 2100^{\circ} \mathrm{C}$ (as measured by an infrared pyrometer) with an estimated $\mathrm{H}_{2} \rightarrow \mathrm{H}^{*}$ conversion efficiency of about 6-7\% for the conditions used (Sutoh et al 1995). Hydrogen doses were measured in terms of molecular hydrogen exposures in kilo-Langmuirs $(\mathrm{kL})\left(1 \mathrm{~kL}=10^{-3}\right.$ torr.s). During AHC, the chamber pressure was typically $3 \times 10^{-5}$ mbar. The distance between the sample and the atomic hydrogen source was approximately $5 \mathrm{~cm}$. Each sample was degassed for one hour to $360^{\circ} \mathrm{C}$ prior to $\mathrm{AHC}$, and annealed to $400^{\circ} \mathrm{C}$ during $\mathrm{AHC}$ by a combination of the atomic hydrogen source filament and the sample filament mounted behind. HREEL spectra were recorded using a range of incident electron energies between 7 and $100 \mathrm{eV}$ with a resolution of 10 meV full width at half maximum (FWHM). XPS spectra were collected to obtain chemical composition and chemical state information before and after $\mathrm{AHC}$, using unmonochromated $\mathrm{Al} \mathrm{K} \mathrm{K}_{\alpha}$ radiation and a $150^{\circ}$ spherical sector analyser, with an overall system resolution of $\sim 1.4 \mathrm{eV}$ for the settings used.

\section{Results and Discussion}

XPS spectra from the as-loaded InAs(110) surface are shown in Figure 1 (dotted lines). The main contribution to the C 1s XPS spectrum indicates the presence of hydrocarbons on the surface. The shoulder on the high binding energy side may be due to the presence of alcohols, carbonates formed after the adsorption of atmospheric $\mathrm{CO}_{2}$, and bicarbonates (Wolan et al 1997). The presence of In-oxide and $\mathrm{As}_{2} \mathrm{O}_{3}$ is indicated by reference to the binding energies of peaks in the In 3d, As 2p and 3d, and O 1s XPS spectra (Moulder et al 1992). The precise nature of the In-oxide could not be identified by XPS because the different oxides are indistinguishable because of the small chemical shifts of the In $3 \mathrm{~d}$ lines - the peak positions are consistent with the presence of $\mathrm{In}_{2} \mathrm{O}_{3}$, the most common In-oxide, and sub-oxides such as $\operatorname{In}_{2} \mathrm{O}$. As there is no component of the As $3 \mathrm{~d}$ spectrum at $\sim 46 \mathrm{eV}$, the presence of the other common As-oxide, $\mathrm{As}_{2} \mathrm{O}_{5}$, above the XPS detection limit is ruled out. This oxide has previously been reported to be unstable in ultra-high vacuum, decomposing into $\mathrm{As}_{2} \mathrm{O}_{3}$ and oxygen within a few hours of formation (Schäfer et al 2000). 

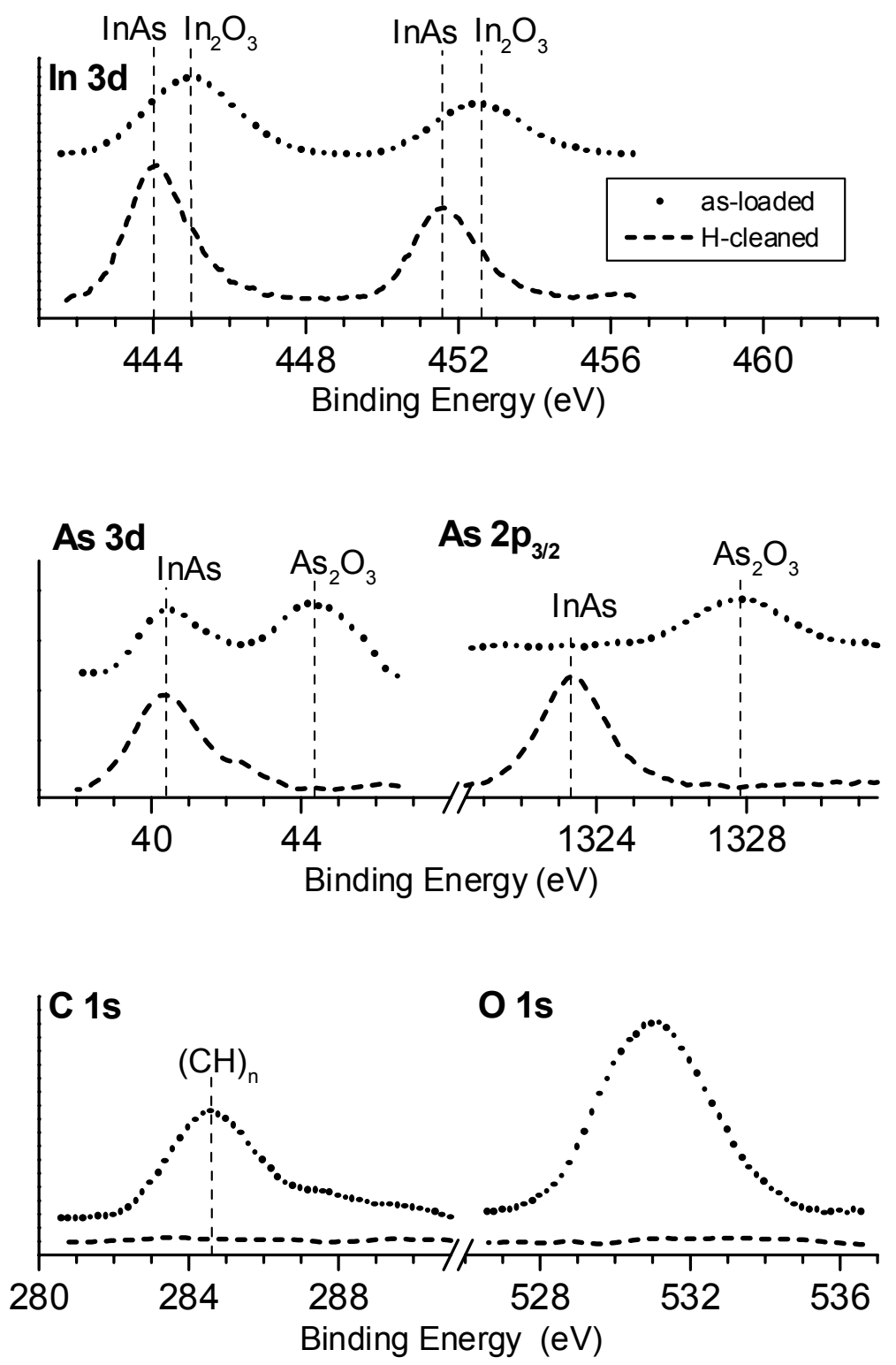

Figure 1. In $3 \mathrm{~d}$, As $3 \mathrm{~d}$, As $2 \mathrm{p}_{3 / 2}, \mathrm{C} 1 \mathrm{~s}$ and $\mathrm{O} 1 \mathrm{~s}$ XPS spectra from as-loaded InAs(110) (dotted lines) and from clean InAs(110) prepared by an AHC dose of $120 \mathrm{~kL}$ with a sample temperature of $400^{\circ} \mathrm{C}$ (dashed lines). All y-axes are in arbitrary units.

An approximate surface composition was determined from the relative intensities of various photoelectron peaks by applying the appropriate atomic sensitivity factor (ASF) for each core level. Errors in determining the surface atomic composition result from the fact that photoelectron lines for the different elements present occur at different binding energies. Consequently, the photoelectrons analysed have different kinetic energies and mean free paths. This means that information contained in the XPS spectra is integrated over different depths for the different elements and makes the assessment of uncertainty in the binding energies difficult. However, from straightforwardly applying the ASF values and using the As 3d line for As because its associated mean free path is closer to that of the In $3 \mathrm{~d}, \mathrm{O} 1 \mathrm{~s}$ and $\mathrm{C} 1 \mathrm{~s}$ photoelectrons than that of the $2 \mathrm{p}_{3 / 2}$ line, the elemental surface composition before cleaning is $47 \% \mathrm{O}, 37 \% \mathrm{C}, 9 \% \mathrm{In}$, and $7 \% \mathrm{As}$.

An estimate of the thickness of the oxide layer was obtained by virtue of the attenuation of the substrate signal by the oxide overlayer. The As $3 \mathrm{~d}$ spectrum is suitable for this analysis as the chemical shift between substrate and oxide peaks is large, allowing the substrate signal alone to be 
examined before and after oxide layer removal. The attenuated signal from the substrate beneath the oxide layer $\mathrm{I}_{\mathrm{s}}$ is given by

$$
\mathrm{I}_{\mathrm{s}}=\mathrm{I}_{\mathrm{s} 0} \exp (-z / \lambda)
$$

where $\mathrm{I}_{\mathrm{s} 0}$ is the unattenuated clean surface signal obtained after AHC, $z$ is the thickness of the oxide layer, and $\lambda$ is the inelastic mean free path of the As $3 \mathrm{~d}$ photoelectrons $27.5 \AA$ (Schaefer et al 1990). Allowing for the error associated with determining the peak areas, this gives an oxide layer thickness of $30 \pm 5 \AA$. This is qualitatively consistent with the information given by comparing the as-loaded spectra of the As $3 \mathrm{~d}$ and As $2 \mathrm{p}_{3 / 2}$ photoelectrons and considering their different inelastic mean free paths. The lower kinetic energy (higher binding energy) As $2 \mathrm{p}_{3 / 2}$ electrons have a mean free path of $\sim 7.5 \AA$ (Schaefer et al 1990), compared with the $\sim 27.5 \AA$ of the higher kinetic energy (lower binding energy) As $3 \mathrm{~d}$ electrons. Because of the exponential attenuation, over $98 \%$ of each signal comes from within four mean free path lengths of the surface. The fact that no As $2 \mathrm{p}_{3 / 2}$ signal from the InAs substrate is visible in the as-loaded spectrum indicates that the oxide layer is at least $30 \AA(4 \times 7.5 \AA)$ thick.

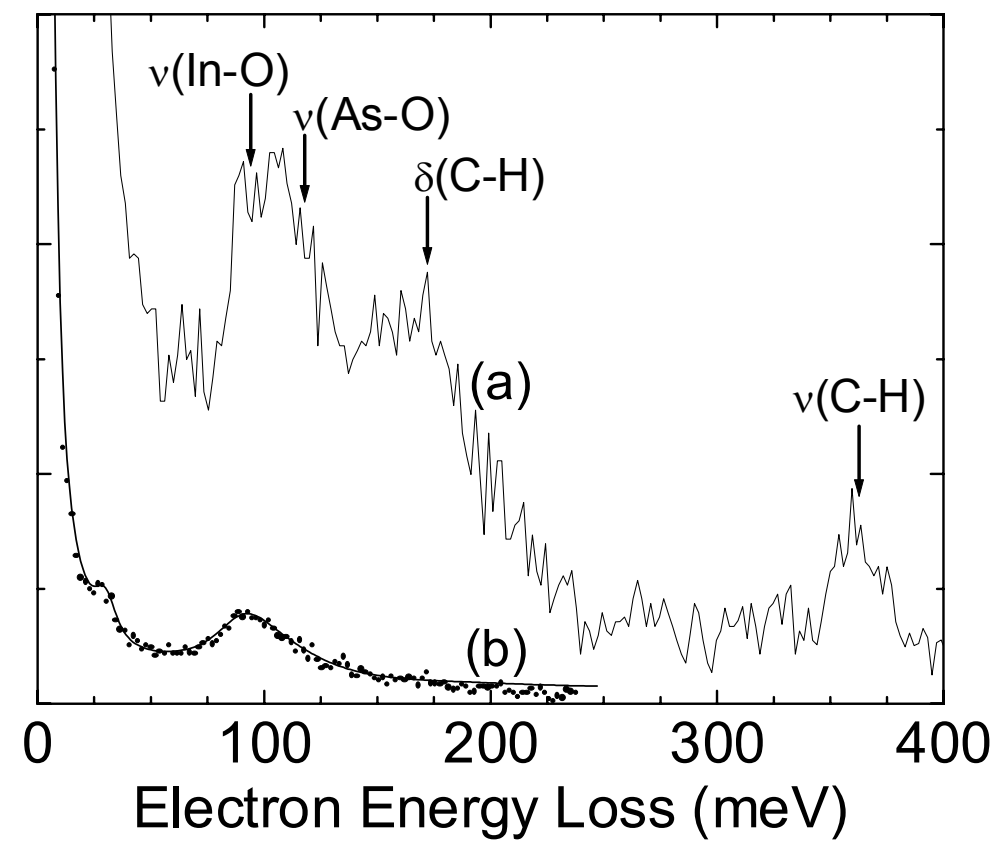

Figure 2. Specular HREEL spectra, recorded at $15 \mathrm{eV}$, with an instrumental broadening of $10 \mathrm{meV}$ FWHM, from (a) an as-loaded InAs(110) sample; (b) after annealing for one hour at $360^{\circ} \mathrm{C}$ and a total of $120 \mathrm{~kL}$ of atomic hydrogen cleaning at a sample temperature of $400^{\circ} \mathrm{C}$. The dotted line of spectrum (b) depicts the experimental points and the solid line is the simulated spectrum calculated using semi-classical dielectric theory.

The as-loaded samples gave a very weak and broad specular elastic peak in HREELS, with an angular spread of $15^{\circ}$ full width at half maximum (FWHM); they exhibited no ordered LEED pattern. Figure 2 (a) shows a specular HREEL spectrum from an as-loaded sample and features a peak at $106 \mathrm{meV}$ - almost twice the width of the elastic peak. This is attributed to a combination of In-O and As-O vibrational modes. These are known to be at $\sim 87$ and $\sim 118 \mathrm{meV}$ respectively (Ibach and Mills et al 1982). However, it is apparent that the broad peak at $106 \mathrm{meV}$ in spectrum (a) of Figure 2 cannot be solely due to the combination of two symmetric peaks centred at 87 and $118 \mathrm{meV}$. However, when the character of an intramolecular bond changes, relatively large frequency shifts can occur (Ibach and Mills et al 1982). It is therefore suggested that the origin of the low loss energy side of this peak is In-O vibrational modes of several different frequencies in 


\section{SURFACE PREPARATION OF InAn(110) USING ATOMIC HYDROGEN}

the range 87 to $\sim 100 \mathrm{meV}$ resulting from the various intramolecular bonds present in the different In-oxides likely to exist in the native oxide, including $\operatorname{In}_{2} \mathrm{O}_{3}$ and suboxides such as $\operatorname{In}_{2} \mathrm{O}$. The peak centred at $\sim 170 \mathrm{meV}$ on the HREEL spectrum recorded from the as-loaded InAs (100) surface is due to the combined hydrocarbon deformation modes, $\delta(\mathrm{C}-\mathrm{H})$ (Aquino et al 1995), whilst the peak at $360 \mathrm{meV}$ is attributed to the well known hydrocarbon stretching mode, $v(\mathrm{C}-\mathrm{H})$ (Ibach and Mills et al 1982). The as-laoded spectrum is quite noisy and has low resolution because of the disordered nature of the atmospheric contamination present. As a result, the presence of alcohols and carbonates suggested by the $\mathrm{C}$ 1s XPS spectrum cannot be unambiguously confirmed because their vibrational modes occur at around the same part of the spectrum as the $\delta(\mathrm{C}-\mathrm{H})$ modes (Jones et al 1989). The intensity of all the peaks in the as-loaded spectrum of Figure 2 decreased as the incident electron energy was increased, confirming that they are due to vibrational modes and not collective excitations.

A clean surface was obtained after a total of $120 \mathrm{~kL}$ of AHC. The XPS spectra recorded from the clean surface are shown in Figure 1 (dotted lines). As shown in this figure, the $\mathrm{C} 1 \mathrm{~s}$ and $\mathrm{O} 1 \mathrm{~s}$ signals were below the detection limit of XPS. The binding energy of the In $3 \mathrm{~d}$ lines has shifted down by $1 \mathrm{eV}$, indicative of the removal of In oxide and the presence of InAs at the surface. The $\mathrm{As}_{2} \mathrm{O}_{3}$ peaks in the As $3 \mathrm{~d}$ and As $2 \mathrm{p}_{3 / 2}$ spectral regions disappeared, with the InAs peak becoming stronger in the As $3 \mathrm{~d}$ spectrum and appearing for the first time in the As $2 \mathrm{p}_{3 / 2}$ region. Bearing in mind the caveats mentioned earlier with regard to determination of surface atomic composition using photoemission results, these XPS spectra suggest that the clean surface consists of $53 \%$ In and $47 \%$ As, close to the 1:1 stoichiometry expected for the non-polar (110) surface.

In the case of $\mathrm{GaAs}(100)$, it has been proposed that the removal of $\mathrm{As}_{2} \mathrm{O}_{3}$ occurs by the formation and desorption of $\mathrm{AsH}_{3}$, whilst the oxygen generated in this process reacts with surface gallium to form more $\mathrm{Ga}_{2} \mathrm{O}_{3}$ (Mikhailov et al 1992). A similar mechanism is proposed here with the formation of more $\operatorname{In}_{2} \mathrm{O}_{3}$ which is corroborated by vibrational spectroscopy results (Veal and McConville, 2000). The proposed reaction for $\mathrm{As}_{2} \mathrm{O}_{3}$ removal and creation of additional $\operatorname{In}_{2} \mathrm{O}_{3}$ is

$$
\mathrm{As}_{2} \mathrm{O}_{3}+2 \mathrm{InAs}+12 \mathrm{H}^{*} \rightarrow \mathrm{In}_{2} \mathrm{O}_{3}+4 \mathrm{AsH}_{3} \uparrow,
$$

where $\mathrm{H}^{*}$ represents thermally excited hydrogen atoms and $\uparrow$ indicates the desorption of the reaction product. Upon further AHC treatment, it is suggested that the $\operatorname{In}_{2} \mathrm{O}_{3}$ is eventually removed, by analogy with the case of $\mathrm{Ga}_{2} \mathrm{O}_{3}$ (Mikhailov et al 1992, Yamada et al 1993), according to the reaction

$$
\mathrm{In}_{2} \mathrm{O}_{3}+4 \mathrm{H}^{*} \rightarrow \mathrm{In}_{2} \mathrm{O} \uparrow+2 \mathrm{H}_{2} \mathrm{O} \uparrow
$$

The clean surface exhibited the clear, sharp $(1 \times 1)$ LEED patterns shown in Figure 3 with the rectangular spacing of diffraction spots characteristic of the (110) surface. Increased surface ordering was also apparent from the more intense specular elastic peak in HREELS, with a much reduced angular spread ( $\left.3^{\circ} \mathrm{FWHM}\right)$. An HREEL spectrum recorded from the clean surface is shown in Figure 2. Whilst the clean surface spectrum shown does not extend to the loss energies of all the contaminant vibrational modes, it was independently confirmed that their intensities had been reduced to below the detection limit. A series of normalised specular HREEL spectra were recorded with a range of incident electron energies from a clean $\operatorname{InAs}(110)$ surface prepared by annealing at $360^{\circ} \mathrm{C}$ followed by a $120 \mathrm{~kL}$ dose of $\mathrm{AHC}$ with a sample temperature of $400^{\circ} \mathrm{C}$. The peak at $\sim 90 \mathrm{meV}$ in all the spectra is due to the conduction-band electron plasmon excitation. The width of this feature decreases at higher incident electron energies, whilst its intensity increases relative to the elastic peak. As the incidence energy is reduced, the plasmon peak becomes more asymmetric and less well defined on the high loss energy side; a small shift towards higher loss energy also occurs. The shoulder on the elastic peak at $\sim 29 \mathrm{meV}$ is assigned to Fuchs-Kliewer surface optical phonon excitations (Mönch et al 1993): this feature does not change in loss energy, 
but its intensity decays rapidly with increasing incidence energy, disappearing completely above 60 $\mathrm{eV}$.

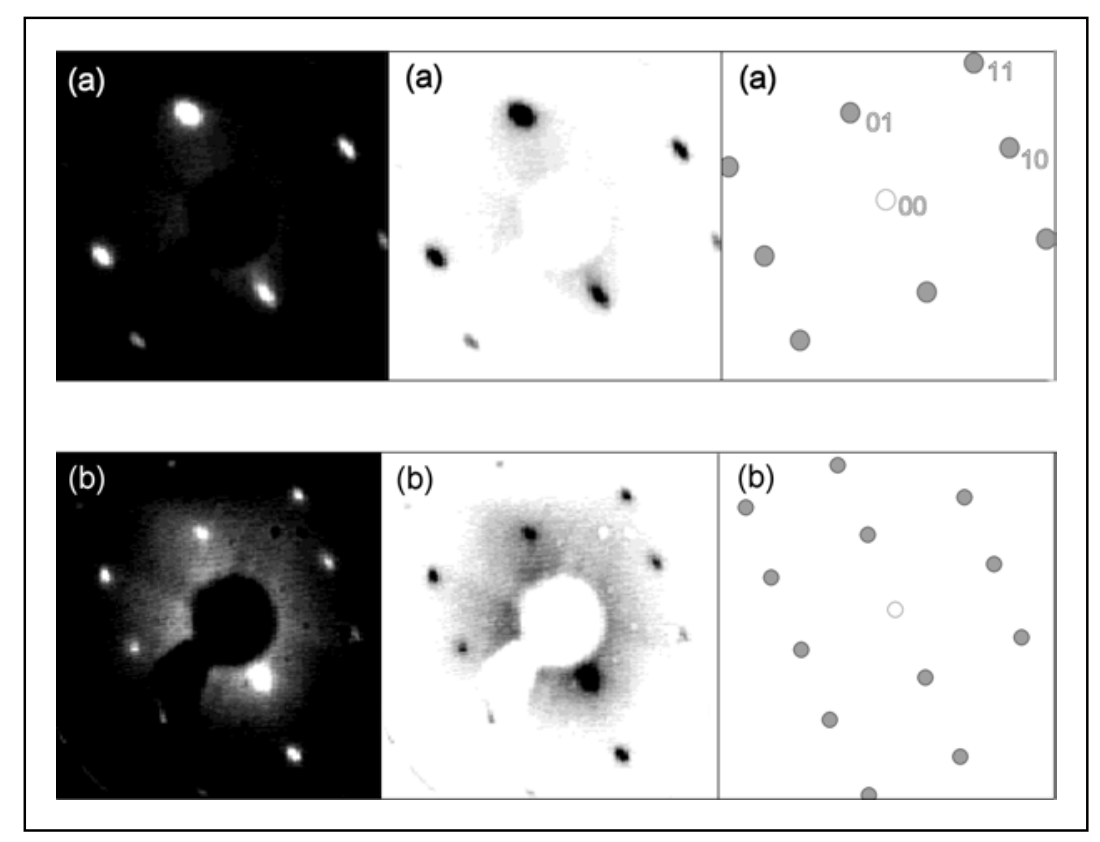

Figure 3. (1×1) LEED patterns (left) recorded using beam energies of (a) $31 \mathrm{eV}$ and (b) $49 \mathrm{eV}$ from InAs(110) prepared by an AHC dose of $120 \mathrm{~kL}$ with a sample temperature of $400^{\circ} \mathrm{C}$. The inverted images (centre) and schematic diagrams (right) are also shown. The diffraction spots obscured by the electron gun are shown unshaded in the schematic pattern.

The HREEL spectra of the atomic hydrogen cleaned surface were analysed using the semiclassical dielectric theory of HREELS (Lambin et al 1990). The plasma dielectric function was based on the hydrodynamic model, with the plasma frequencies, plasmon lifetimes, and spatial dispersion coefficients as parameters (Bell et al 1996b). A three-layer model was used to simulate the experimental spectra across the entire incident-electron energy range, consisting of a plasmonfree surface "dead" layer, accumulation layer, and a semi-infinite bulk. Below the $35 \AA$ dead layer, the $55 \AA$ accumulation layer had a carrier concentration of $6.7 \times 10^{18} \mathrm{~cm}^{-3}$, above a semi-infinite bulk with a carrier concentration of $3.9 \times 10^{18} \mathrm{~cm}^{-3}$ : that is, unchanged from the nominal bulk doping value given by the sample manufacturer. The band bending was estimated to be $115 \mathrm{meV}$, with a triangular potential well width of $140 \AA$, giving a surface charge density of $6.3 \pm 2.0 \times 10^{11}$ $\mathrm{cm}^{-2}$. This is in agreement with the values of $10^{11}-10^{12} \mathrm{~cm}^{-2}$ found on other faces of InAs (Olsson et al 1996, Bell et al 1997) and on hydrogen terminated InAs(110) (Chen et al 1989).

The spatial dispersion coefficient for the simulations was calculated using the Thomas-Fermi model (TFM), giving a value of $0.43 \times 10^{6} \mathrm{~ms}^{-1}$, which successfully reproduced the observed plasmon peak dispersion in the experimental spectra. This indicates that the hydrogen cleaning treatment does not cause subsurface damage: for ion bombarded and annealed samples spatial dispersion coefficients are significantly lower than those predicted by the TFM because of additional defect scattering (Bell et al 1996b). The carrier mobility of AHC InAs(110) deduced from the plasmon lifetimes used in the simulations is five times higher than for samples from the same wafer subjected to grazing incidence $500 \mathrm{eV}$ IBA (Veal and McConville 2001), providing further evidence in favour of $\mathrm{AHC}$ as a surface preparation technique.

An accumulation layer has also been observed on InAs(110) cleavage surfaces after good mirror-like cleavages, as seen by visual inspection and LEED (Karlsson et al 1998). Whilst an "ideal" cleavage gives a flat band condition on the InAs(110) surface with no electron accumulation, the surface electronic structure is extremely sensitive to the presence of contamination and defects, resulting in downward band bending and accumulation layer formation. 


\section{SURFACE PREPARATION OF InAn(110) USING ATOMIC HYDROGEN}

From cleave to cleave large changes in step densities, and consequently adsorption properties and defect densities occur, resulting in significant variations in surface properties. Atomic hydrogen cleaning is an effective alternative method of obtaining clean, undamaged $\operatorname{InAs}(110)$ surfaces prior to further in situ processing.

In conclusion, it has been demonstrated that clean, ordered InAs(110) surfaces can be obtained by exposing the atmospherically contaminated substrates to atomic hydrogen at $400^{\circ} \mathrm{C}$ with a molecular hydrogen dose of $120 \mathrm{~kL}$. During atomic hydrogen cleaning, the $\mathrm{As}_{2} \mathrm{O}_{3}$, In-oxides and hydrocarbons are removed completely. Furthermore, this process produces no detectable damage: dielectric theory simulations of the plasmon excitations in the HREEL spectra indicate carrier mobilities and spatial dispersion coefficients significantly higher than those of samples prepared by IBA; and below the accumulation layer the carrier concentration was found to be that of the nominal S-doping.

\section{References}

AQUINO, A. A., HILL, J. J. and JONES, T. S. 1995. Evidence for a surface methylene species in the decomposition of trimethylgallium on $\operatorname{GaAs}(100)-(4 \times 1)$ : a high resolution electron energy loss spectroscopy study. Surf. Sci. 327: 74-80.

BELL, G.R., MCCONVILLE, C.F. and JONES, T.S. 1996a. Plasma excitations and the effects of surface preparation in n-type $\operatorname{InAs}(001)$ studied by electron energy loss spectroscopy. Appl. Surf. Sci. 104/105: 17-23.

BELL, G.R., MCCONVILLE, C.F. and JONES, T.S. 1996b. Plasma excitations and accumulation layers in heavily doped InAs(001). Phys. Rev. B 54: 2654-2661.

BELL, G.R., JONES, T.S. and MCCONVILLE, C.F. 1997. Accumulation layer profiles at InAs polar surfaces. Appl. Phys. Lett. 71: 3688-3690.

BELL, G.R., KAIJAKS, N.S., DIXON, R.J. and MCCONVILLE, C.F. 1998. Atomic hydrogen cleaning of polar III-V semiconductor surfaces. Surf. Sci. 401: 125-137.

CHEN, Y., HERMANSON J.C. and LAPEYRE, G.J. 1989. Coupled plasmon and phonon in the accumulation layer of InAs(110) cleaved surfaces. Phys. Rev. B 39: 12682-12687.

GOTO, S., YAMADA, M. and NOMURA, Y. 1995. Surface cleaning of Si doped/undoped GaAs substrates. Jpn. J. Appl. Phys. 234: L1180-L1183.

IBACH, H. and MILLS, D.L. 1982. Electron Energy Loss Spectroscopy and Surface Vibrations, Academic, New York.

JONES, T.S., ASHTON, M.R., RICHARDSON, N.V. and MCCONVILLE, C.F. 1989. Electron energy-loss studies of InSb (100). J. Phys.: Condens. Matter 1: SB207-SB208.

KARLSSON, H.S., VISELGA, R. and KARLSSON, U.O. 1998. Electron accumulation at the InAs(110) cleavage surface. Title. Surf. Sci. 402-404: 822-826.

KIKAWA, T., OCHIAI, I. and TAKATANI, S. 1994. Atomic hydrogen cleaning of GaAs and InP surfaces studied by photoemission spectroscopy. Surf. Sci. 316: 238-46.

LAMBIN, Ph., VIGNERON, J. and LUCAS, A.A. 1990. Computation of the surface electronenergy-loss spectrum in specular geometry for an arbitrary plane-stratified medium. Computer Phys. Commun. 60: 351-64.

LIANG, Y., PACKARD, W.E., DOW, J.D., HO, H. and LAPEYRE, G.J. 1993. Monatomic steps on the InAs(110) surface. Phys. Rev. B 48: 11942-11945.

MAGNEE, P.H.C., DEN HARTOG, S.G., VAN WEES, B.J., KLAPWIJK, T.M., VAN DE GRAAF, W. and BORGHS, G. 1995. Influence of low-energy Ar-sputtering on the electronicproperties of InAs-based quantum-well structures. Appl. Phys. Lett. 67: 3569-3571.

MIKHAILOV, G.M., BUKIN, P.V., KHUDOBIN, S.A., CHUMAKOV, A.A. and SHAPOVAL, S.Y. 1992. XPS investigation of the interaction between ECR-excited hydrogen and the native oxide of GaAs(100). Vacuum 43: 199-201.

MONCH, W. 1993. Semiconductor surfaces and interfaces. Springer, Berlin. 
MOULDET, J.F., STICKLE, W.F., SOBOL P.E. and BOMBEN, K.D. 1992. Handbook of X-ray Photoelectron Spectroscopy. ed. J. Chastain. Perkin-Elmer Corporation, Eden Prairie, MN.

OLSSON, L.O., ANDERSSON, C.B.M., HAKANSSON, M.C., KANSKI, J., ILVER, L. and KARLSSON, U.O. 1996. Charge accumulation at InAs surfaces. Phys. Rev. Lett. 76: 36263629.

PETIT, E.J., HOUZAY, F. and MOISON, J.M. 1992. Interaction of atomic hydrogen with native oxides on InP(100). Surf. Sci. 269/270: 902-908.

PETIT, E.J. and HOUZAY, F. 1994. Optimal surface cleaning of GaAs(001) with atomic hydrogen. J. Vac. Sci. Technol. B 12: 547-550.

SCHAFER, M., NAUMANN, W., FINNBERG, T., HANNSS, M., DUTSCHKE, A., and ANTON, R. 2000. UV/ozone-activated growth of oxide layers on $\operatorname{InAs}(001)$ surfaces and oxide desorption under arsenic pressure. Appl. Surf. Sci., 158: 147-158.

SCHAEFER, J.A., PERSCH, V., STOCK, S., ALLINGER, TH. and GOLDMANN, A. 1990. Etching of GaAs(100) by activated hydrogen. Europhys. Lett. 12: 563-568.

SUTOH, A., OKADA, Y., and KAWABE, M. 1995. Cracking efficiency of hydrogen with tungsten filament in molecular beam epitaxy. Jpn. J. Appl. Phys. 234: L1379-L1382.

VEAL, T.D. and MCCONVILLE, C.F. 2000. Controlled oxide removal for the preparation of damage-free InAs(110) surfaces. Appl. Phys. Lett. 77: 1665-1667.

VEAL, T.D. and MCCONVILLE, C.F. 2001. Profiling of electron accumulation layers in the nearsurface region of InAs(110). Phys. Rev. B 64: 085311.

WOLAN, J.T., MOUNT, C.K. and HOFLUND, G.B. 1997. Chemical reactions induced by the room temperature interaction of hyperthermal atomic hydrogen with the native oxide layer on $\mathrm{GaAs}(001)$ surfaces studied by ion scattering spectroscopy and x-ray photoelectron spectroscopy. J. Vac. Sci. Technol. A 15: 2502-2506.

YAMADA, M., IDE, Y., and TONE, K. 1993. Interaction of atomic-hydrogen with GaAs(001) surface oxide - volatile Ga-oxide formation. Appl. Surf. Sci. 70/71: 531-535. 